ARTICLE

\title{
Higher Order $\alpha$ Mode Eigenvalue Calculation by Monte Carlo Power Iteration
}

\author{
Toshihiro YAMAMOTO* \\ Kyoto University, 2-1010 Asashironishi, Kumatori-cho, Sennan-gun, Osaka, 590-0494, Japan
}

\begin{abstract}
A recently proposed method implementing a pseudo absorption term in the neutron transport equation for $\alpha$ mode eigenvalue calculations in the Monte Carlo technique has been discussed. This method is known to overcome the difficulty in $\alpha$ mode eigenvalue calculations for fissionable systems with large subcriticality. This paper has demonstrated that this technique certainly can provide stable $\alpha$ mode eigenvalue calculations for problems that the original MCNP 4C fails to solve and that the figure of merit of the calculated $\alpha$ value is largely improved. However, caution should be taken that the variance of calculated $\alpha$ values is underestimated due to the inter-cycle correlation of the $\alpha$ values. A method that provides eigenfunctions with higher order criticality eigenvalues has been applied to the second order $\alpha$ mode eigenvalue calculations. The method partitions a whole space into two regions. The estimate of the multiplication factor in each region is forced to be equal to each other. This method is found to be applicable to calculations for the second $\alpha$ mode eigenfunction. The conditions on convergence of the second $\alpha$ mode eigenfunction are discussed. Hotelling's method, which explicitly subtracts lower order eigenfunctions from the fission source distribution, has been applied to a test problem. The third and fourth order forward eigenfunctions are successfully obtained with the use of Hotelling's method.
\end{abstract}

KEYWORDS: Monte Carlo, $\alpha$ mode, higher order mode, criticality calculation, power iteration, eigenvalue, pulsed neutron

\section{Introduction}

A prompt neutron time decay constant (hereafter called $\alpha$ ) is one of important parameters to acquire the neutronic property of a fissionable system. In an experiment that uses a fissionable material, the constant $\alpha$ is one of values that can be directly measured by the pulsed neutron method, Ross- $\alpha$ method, Feynman- $\alpha$ method and so on. On the other hand, an effective neutron multiplication factor $k_{\text {eff }}$ cannot be exactly measured except when the system is critical. Thus, one of the methods of validating criticality calculation codes is the comparison of the calculated $\alpha$ and measured $\alpha$. When validating Monte Carlo criticality calculation codes with $\alpha$ value comparison, the capability of calculating $\alpha$ values is required in Monte Carlo criticality calculation codes. For example, Monte Carlo simulation for pulsed neutron method can provide an $\alpha$ value, which can be available for the code validation. Another method for obtaining an $\alpha$ value by the Monte Carlo criticality calculation method is an $\alpha$ eigenvalue mode calculation. ${ }^{1)}$ The function of $\alpha$ eigenvalue mode calculation had been installed in a continuous energy Monte Carlo code MCNP version 4C. ${ }^{2)}$ The $\alpha$ value obtained by the eigenvalue mode calculation corresponds to a fundamental mode. Like the criticality calculation for a fundamental mode neutron multiplication factor $k_{\text {eff }}$, the $\alpha$ eigenvalue mode calculation is relatively easy as compared to radiation shielding calculations that require elaborate variance reduc-

*Corresponding author, E-mail:pbrasko@yahoo.co.jp tion techniques. The $\alpha$ eigenvalue and the fission source distribution converge toward the fundamental mode without any special treatments.

On the other hand, in the measurement of an $\alpha$ value, the contamination of the measured $\alpha$ value by higher order modes is unavoidable. The effect of the higher order modes depends on the location of a neutron source and neutron detectors. Endo et al. derived generalized but complicated theoretical formulae for the well-known Feynman- $\alpha$ method (i.e., the second order neutron correlation technique) and the third order neutron correlation technique that takes into consideration higher order $\alpha$ mode eigenvalues and eigenfunctions. ${ }^{3)} \mathrm{A}$ desirable technique of the measurement of a fundamental mode $\alpha$ value is to eliminate the higher order mode effects as much as possible. If this elimination is successful, simple formulae for the third order neutron correlation can be applied to measuring a fundamental mode $\alpha$ value. If the contamination of higher order modes is inevitable, generalized but complicated formulae have to be used. Therefore, the knowledge on the higher order $\alpha$ mode eigenvalues and eigenfunctions is required. From these view points, the identification of the $\alpha$ mode higher order mode eigenfunctions and eigenvalues is important for the measurement of an accurate fundamental mode $\alpha$ value as well as the code validation using $\alpha$ values.

Some attempts to calculate eigenfunctions with higher order criticality eigenvalues have been done by Booth ${ }^{4,5)}$ and Yamamoto. ${ }^{6}$ For example, to obtain the second eigenfunction, the method partitions a whole space into two regions. In 
the course of the power iteration of a Monte Carlo criticality calculation, the amplitude of the eigenfunction in each region is adjusted such that the estimate of the eigenvalue in each region is forced to be equal to each other. This technique has an advantage in requiring no information on the lower order eigenfunctions and is suitable for Monte Carlo criticality calculations. Therefore, it is expected to be a matter of course that this technique may be applicable to generate $\alpha$ mode higher order eigenfunctions by Monte Carlo techniques. This paper may be the first attempt to obtain higher order mode $\alpha$ eigenvalues and eigenfunctions by the Monte Carlo technique.

In this paper, the Monte Carlo calculation technique of an $\alpha$ mode eigenvalue is revisited. Recently, a method for improving the reliability of the $\alpha$ mode eigenvalue calculation with Monte Carlo techniques has been proposed. ${ }^{7)}$ The effect of this improvement is discussed. Then, the method for higher order criticality eigenfunctions with Monte Carlo techniques is applied to generating the second order $\alpha$ mode eigenvalue. The conditions on the convergence of the second order eigenfunction are treated. Finally, the prospect of further higher order $\alpha$ mode eigenvalue is discussed.

\section{Review of $\alpha$ Mode Eigenvalue Calculation with Monte Carlo Technique}

\section{Algorithm of $\alpha$ Mode Eigenvalue Calculation in Monte} Carlo Technique

Before discussing the higher order $\alpha$ mode calculation, the fundamental mode calculation for an $\alpha$ eigenvalue with Monte Carlo technique is discussed. A neutron transport equation in the prompt neutron decay constant eigenvalue mode is written as

$$
\begin{aligned}
& \vec{\Omega} \cdot \nabla \phi(r, \vec{\Omega}, E)+\Sigma_{t}(r, E) \phi(r, \vec{\Omega}, E)= \\
& \int d \vec{\Omega}^{\prime} \int d E^{\prime} \phi\left(r, \vec{\Omega}^{\prime}, E^{\prime}\right) \Sigma_{s}\left(r, \vec{\Omega}^{\prime} \rightarrow \vec{\Omega}, E^{\prime} \rightarrow E\right) \\
& +\frac{\chi_{p}(E)}{4 \pi} \int d \vec{\Omega}^{\prime} \int d E^{\prime} \phi\left(r, \vec{\Omega}^{\prime}, E^{\prime}\right) v_{p} \Sigma_{f}\left(r, E^{\prime}\right) \\
& +\alpha \phi(r, \vec{\Omega}, E) / v(E),
\end{aligned}
$$

where $r$ is a three-dimensional position and $v(E)$ is a neutron velocity with energy $E$. The subscript $p$ stands for the value of prompt neutrons. Other notations are standard in neutronics. In this transport equation for a subcritical system, the fundamental mode neutron flux changes exponentially with the fundamental mode eigenvalue (i.e., time-decay constant) $\alpha(>0)$ after higher order modes die out as

$$
\phi(r, \vec{\Omega}, E, t) \propto e^{-\alpha t} .
$$

If a fissionable system is supercritical without delayed neutrons, the neutron flux grows exponentially with the time-decay constant $\alpha(<0)$. In a subcritical fissionable system, the last term in Eq. (1) plays a role as a source term to make the eigenvalue mode equation hold stable. Yamamoto et al. adopted a following different approach for implementing the last term in Eq. (1) during the random walk of Monte Carlo calculations. ${ }^{8}$ The existence of the last term in Eq. (1) shows that neutron weight increase or decrease when a neutron flies through a medium. If the neutron flux in the last term in Eq. (1) is replaced by the neutron weight, the term represents the weight change per unit particle flight distance. Thus, the weight change $d W$ of a neutron particle that flies by an infinitesimal length of $d s$ is given by

$$
d W=\alpha / v(E) \cdot W d s .
$$

Here, suppose that the $\alpha$ value is known from the calculation in the previous cycle of a power iteration. After the flight of a length $s$, an initial weight $W_{0}$ is changed to $W$ as

$$
W=W_{0} \exp (\alpha s / v(E)) .
$$

$T_{i}$, the product of the track length and the weight in the $i$-th flight with a length $s_{i}$, is defined by

$$
\begin{aligned}
& T_{i}=\int_{0}^{s_{i}} W_{0} \exp \left(\alpha s_{i}{ }^{\prime} / v_{i}(E)\right) d s_{i}{ }^{\prime} \\
& \left.=W_{0}\left(\exp \left(\alpha s_{i} / v_{i}(E)\right)\right)-1\right) /\left(\alpha / v_{i}(E)\right)
\end{aligned}
$$

where $v_{i}(E)$ is the neutron velocity of the $i$-th flight. The number of fission neutrons $n$ used for the starting source neutrons in the next cycle is determined by

$$
n=I N T\left(W \bar{v}_{p}\left(\Sigma_{f} / \Sigma_{t}\right)+\xi\right)
$$

where $\xi$ is a uniform pseudorandom number between 0 and 1, and INT stands for the integer part of the value within the parentheses. Note that the second term in the parentheses in Eq. (6) is not divided by $k_{\text {eff }}$ unlike criticality calculations.

The integration of two terms on the left hand side in Eq. (1) over all phase space throughout one cycle calculation is equal to total loss of neutrons due to absorption and escape. Thus, the integration is given by the sum of the total weight of starting source particles and increase in weight due to Eq. (3). The scattering term in Eq. (1) does not contribute to the weight change. By integrating the last two terms in Eq. (1) over all phase space throughout one cycle and solving for $\alpha$, an eigenvalue $\alpha^{\prime}$ is estimated as

$$
\alpha^{\prime}=\left(N+\sum_{i} \Delta W_{i}-\sum_{i} v_{p} \Sigma_{f} T_{i}\right) / \sum_{i} T_{i} / v_{i}(E)
$$

where

$i$ is summed over all track lengths in a cycle,

$N=$ number of source histories per cycle,

$$
\Delta W_{i}=W_{0}\left(\exp \left(\alpha s_{i} / v_{i}(E)\right)-1\right) .
$$

Using Eqs. (5) and (8), Eq. (7) is rewritten as

$$
\alpha^{\prime}=\alpha\left(1+\frac{1}{\sum_{i} \Delta W_{i}}\left(N-\sum_{i} v_{p} \Sigma_{f} T_{i}\right)\right)
$$

Then, the next cycle calculation uses $\alpha^{\prime}$ for the $\alpha$ value in Eqs. (4) and (5). A final solution of $\alpha$ eigenvalue is given by simply averaging the $\alpha^{\prime}$ values of all active cycles after discarding some initial cycles that may be contaminated by not fully converged fission source distribution. 
For a fissionable system with a large subcriticality, the last term in Eq. (1) (i.e., source term) becomes more dominant than the second last term in Eq. (1) (i.e., fission term) since the large subcritical system has a large $\alpha$ value. In such a case, the neutron weight becomes too large to keep sustainable Monte Carlo power iteration. ${ }^{9)}$ Abnormal terminations in $\alpha$ mode eigenvalue calculations were reported in the original MCNP version 4C. ${ }^{7)}$ To make $\alpha$ mode eigenvalue calculations more stable, Yen et al. introduced a pseudo neutron absorption term in the $\alpha$ eigenvalue mode neutron transport equation as

$$
\begin{aligned}
& \vec{\Omega} \cdot \nabla \phi(r, \vec{\Omega}, E)+\Sigma_{t}^{*}(r, E) \phi(r, \vec{\Omega}, E)= \\
& \int d \vec{\Omega}^{\prime} \int d E^{\prime} \phi\left(r, \vec{\Omega}^{\prime}, E^{\prime}\right) \Sigma_{s}\left(r, \vec{\Omega}^{\prime} \rightarrow \vec{\Omega}, E^{\prime} \rightarrow E\right) \\
& +\frac{\chi_{p}(E)}{4 \pi} \int d \vec{\Omega}^{\prime} \int d E^{\prime} \phi\left(r, \vec{\Omega}^{\prime}, E^{\prime}\right) v_{p} \Sigma_{f}\left(r, E^{\prime}\right) \\
& +(1+\lambda) \alpha \phi(r, \vec{\Omega}, E) / v(E),
\end{aligned}
$$

where

$$
\Sigma_{t}^{*}(r, E)=\Sigma_{t}(r, E)+\lambda \alpha / v(E) .
$$

The term $\lambda \alpha / v(E)$ in Eq. (11) increases the macroscopic total cross sections, leading to increase in neutron absorption. A parameter $\lambda$ is appropriately adjusted so that the $\alpha$ eigenvalue mode calculation can be stable. The pseudo absorption term can be implemented by replacing $\alpha$ by $(1+\lambda) \alpha$ in Eqs. (4), (5) and (8). Equation (7) is still available for obtaining the $\alpha$ for the next cycle calculation even when the pseudo absorption term is introduced. The last term in the numerator in Eq. (9) is almost equal to $N$, the number of source histories per cycle, regardless of $\lambda$ after the power iteration enters a stable cycle. On the other hand, the denominator in Eq. (9) increases with increasing $\lambda$. Therefore, as seen in Eq. (9), the change in the $\alpha$ value (i.e., $\alpha^{\prime}-\alpha$ ) in each iteration is suppressed by introducing the pseudo absorption term. The effect of the pseudo absorption term is shown below.

\section{Examples of $\alpha$ Mode Eigenvalue Calculations in Monte \\ Carlo Technique}

As numerical tests for the algorithm of $\alpha$ mode eigenvalue calculations explained above, $\alpha$ mode eigenvalue calculations were performed for a solution fuel of enriched uranyl nitrate that was used in the Static Criticality Experimental Facility (STACY) at the Japan Atomic Energy Agency. ${ }^{10)}$ Although the experiments in Ref. 10) were performed for 9.97-wt.\%-enriched uranyl nitrate solution, the ${ }^{235} \mathrm{U}$ enrichment for the test calculations was approximately $6 \mathrm{wt} . \%$ that was used for the experiment campaign of the STACY in 2004. The uranium concentration was approximately $378 \mathrm{~g} / \ell$. The experimental core configuration for the numerical tests was an unreflected cylindrical form with the diameter of $79 \mathrm{~cm}$ and the height of approximately $55 \mathrm{~cm}$. The solution fuel was contained in a cylindrical core tank made of stainless steel. The $k_{\text {eff }}$ of the fuel solution was approximately 0.960 .

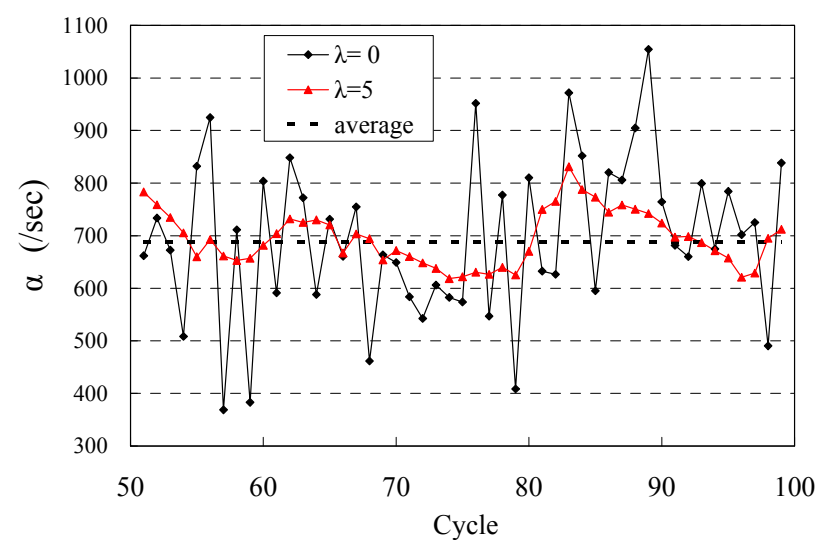

Fig. 1 Calculated $\alpha$ value transitions with and without pseudo absorption term

The algorithm explained above was implemented into MCNP 4C. The $\alpha$ mode eigenvalue calculations for the cylindrical fuel solution system were performed with 25 inactive cycles and 1,000 active cycles at 10,000 neutrons per cycle. The calculated $\alpha$ values, their standard deviations and the relative figure of merit (FOM) for several adjustment parameters $\lambda$ are listed in Table 1 . The $k_{\text {eff }}$ 's, which would be exactly unity in the $\alpha$ mode eigenvalue calculations, are also listed in Table 1 . The FOM are defined as $1 / T \sigma^{2}$, where $T$ is the CPU time and $\sigma$ is the standard deviation. Note that these $\alpha$ values and their standard deviations are the results of simple statistical processing over 1,000 active cycles. Table 1 shows that the standard deviation decreases as the parameter $\lambda$ increases. Figure 1 shows the transitions of the calculated $\alpha$ values in each cycle for several $\lambda$ values. As shown in Fig. 1, the fluctuation of calculated $\alpha$ values becomes suppressed as the parameter $\lambda$ increases. Furthermore, an apparent correlation of $\alpha$ values between neighboring cycles is seen for larger $\lambda$. In other words, the $\alpha^{\prime}$ value calculated by Eq. (7) is influenced by the $\alpha$ value that is used in Eqs. (4) and (5). In the algorithm of this paper, the $\alpha$ value of Eqs. (4) and (5) in the next cycle uses the $\alpha^{\prime}$ value obtained in the previous cycle. Under such a situation with the inter-cycle correlation, simple statistical processing of $\alpha$ values underestimates the standard deviation. This is a common problem arising in criticality calculations that always entail the inter-cycle correlation of fission source distribution. How to estimate an $\alpha$ value used for the next cycle and how to estimate the true standard deviation would be a research issue in the future. However, it is certain that introducing the pseudo absorption term in the $\alpha$ eigenvalue mode neutron transport equation makes $\alpha$ mode eigenvalue calculations stable and reliable.

To verify the $\alpha$ values calculated by the $\alpha$ eigenvalue mode, an equivalent $\alpha$ value was obtained by a different calculation technique using the same Monte Carlo code (MCNP 4C) and the same cross section library (JENDL-3.3). As one of the techniques for the verification, the simulation of the pulsed neutron experiment was performed. Pulsed fission neutrons were generated uniformly within the whole fuel region, and then the subsequent neutron counts were accumulated in time bins. The decay constant $\alpha$, which is 
Table 1 Calculated $\alpha$ values of 6-wt.\%-enriched uranyl nitrate solution for several $\lambda$ 's

\begin{tabular}{|c|c|c|c|c|c|}
\hline & $\lambda$ & $\alpha\left(\mathrm{s}^{-1}\right)$ & $\begin{array}{l}\text { Relative } \\
\text { FOM of } \alpha\end{array}$ & $\sum_{i} \Delta W_{i}$ & $k_{\text {eff }}$ \\
\hline \multirow{3}{*}{$\begin{array}{l}\alpha \text { mode eigenvalue } \\
\text { calculation }\end{array}$} & 0.0 & $689.1 \pm 5.5$ & 1.0 & $398.8 \pm 3.2$ & $1.00010 \pm 0.00042$ \\
\hline & 2.0 & $689.5 \pm 2.3$ & 5.6 & $1177.7 \pm 4.1$ & $1.00016 \pm 0.00024$ \\
\hline & 5.0 & $691.9 \pm 1.6$ & 11.1 & $2376.4 \pm 6.0$ & $1.00017 \pm 0.00021$ \\
\hline $\begin{array}{l}\text { Pulsed neutron me- } \\
\text { thod simulation }\end{array}$ & - & $689.0 \pm 0.2$ & - & & - \\
\hline
\end{tabular}

Table 2 Calculated $\alpha$ values of 4 wt. $\%$ enriched uranium solution for several $\lambda$ 's

\begin{tabular}{|c|c|c|c|c|c|}
\hline & $\lambda$ & $\alpha\left(\mathrm{s}^{-1}\right)$ & $\begin{array}{l}\text { Relative } \\
\text { FOM of } \alpha\end{array}$ & $\sum_{i} \Delta W_{i}$ & $k_{\text {eff }}$ \\
\hline \multirow{3}{*}{$\begin{array}{l}\alpha \text { mode eigenvalue } \\
\text { calculation }\end{array}$} & 2.0 & $4168.4 \pm 1.5$ & 1.0 & $20060 \pm 18$ & $1.00035 \pm 0.00067$ \\
\hline & 3.5 & $4168.5 \pm 0.8$ & 3.5 & $30080 \pm 19$ & $0.99995 \pm 0.00033$ \\
\hline & 5.0 & $4170.2 \pm 0.7$ & 4.8 & $40115 \pm 20$ & $1.00001 \pm 0.00028$ \\
\hline $\begin{array}{l}\text { Pulsed neutron me- } \\
\text { thod simulation }\end{array}$ & - & $4171.2 \pm 0.8$ & - & - & - \\
\hline
\end{tabular}

deemed to be equivalent to the $\alpha$ mode eigenvalue, was obtained by fitting the neutron time-decay in the duration when the higher order mode fluxes apparently decay. The $\alpha$ value obtained by the pulsed neutron simulation is also listed in Table 1 . The $\alpha$ values obtained by the different $\lambda$ values and the pulsed neutron experiment simulation indicate agreement with each other within the statistical uncertainties.

This test problem is a moderate subcritical system that MCNP 4C can successfully yield a converged $\alpha$ eigenvalue. In another test problem, the ${ }^{235} \mathrm{U}$ enrichment and the uranium concentration were reduced to $4 \mathrm{wt} . \%$ and $200 \mathrm{~g} / \ell$, respectively. The height of the cylindrical fuel solution was set to $35 \mathrm{~cm}$ with other conditions being identical to the previous test problem. The $k_{\mathrm{eff}}$ of this fuel solution was approximately 0.537. An $\alpha$ mode eigenvalue calculation by the original MCNP 4C abnormally halted before all cycles to be done were completed. The modified MCNP 4C with the adjustment parameter $\lambda=0$ also failed to keep a sustainable power iteration of $\alpha$ mode eigenvalue. The parameter $\lambda$ was adjusted so that the $\alpha$ mode eigenvalue calculations were normally terminated. Table 2 also shows the results with several $\lambda$ values for the large subcritical system. In this system, the calculations were found to be successfully finished when the parameter $\lambda$ was larger than 2.0. Again, Fig. 2 shows the transitions of the calculated $\alpha$ values in each cycle for several $\lambda$ 's. Even for the smallest $\lambda$, the $\alpha$ values oscillate around the average $\alpha$ value with a longer period as opposed to the case with $\lambda=0$ in Fig. 1. Thus, a certain level of inter-cycle correlation of an $\alpha$ value is inevitable for a large subcritical fissionable system. The simulation of the pulsed neutron experiment was also performed for this large subcritical system. In the same manner as the previous test problem, the time-decay constant was obtained and is listed in Table 2. The $\alpha$ values in Table 2 agree well with each other regardless of the parameter $\lambda$ and the calculation methods.

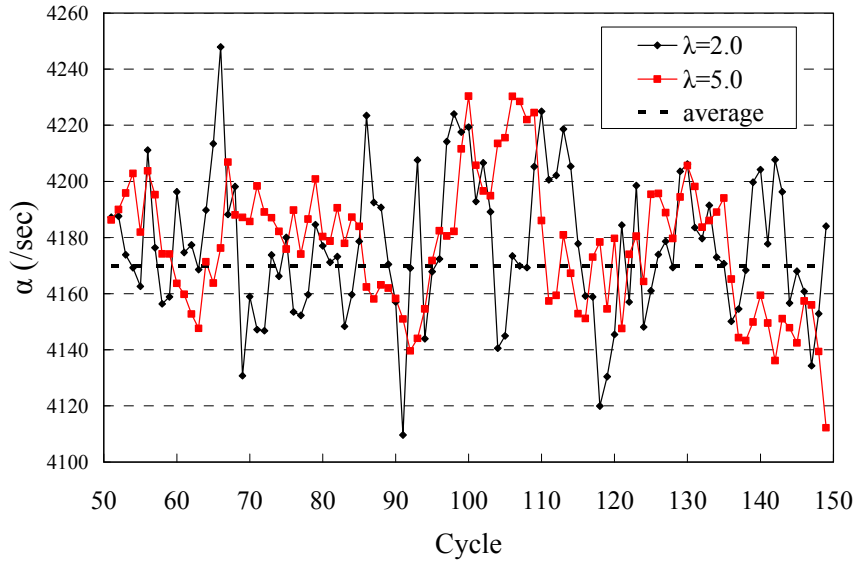

Fig. 2 Calculated $\alpha$ value transitions for $\lambda=2$ and 5

\section{III. $\alpha$ Mode Higher Order Eigenvalue Calculation in Monte Carlo Technique}

\section{Convergence of Fundamental $\alpha$ Mode Eigenvalue}

In this section, higher order $\alpha$ mode eigenvalue calculations using the power iteration method are discussed. Higher order mode calculations usually require subtraction of lower order eigenfunctions from the fission source distribution as represented by Hotelling's method. ${ }^{11,12)}$ Monte Carlo methods, however, have difficulties to make subtraction since Monte Carlo methods define the neutron flux distribution by many particles being randomly distributed throughout a fissionable system.

Some attempts to perform higher order criticality eigenvalues without subtraction technique have been done by Booth $^{4,5)}$ and Yamamoto. ${ }^{6)}$ This technique is suitable for Monte Carlo calculations. This paper attempts to apply the technique to higher order $\alpha$ mode eigenvalue calculations.

In the power iteration method for a fundamental mode $\alpha$ eigenvalue calculation, the initial fission source distribution, 
which may be far from the fundamental $\alpha$ mode, is given at the beginning of the calculation. The ratio of a higher order eigenfunction's amplitude to the fundamental mode's one attenuates at each power iteration, then the fission source distribution converges to the fundamental mode. Let the fission source distribution and the $i$-th mode eigenfunction be $P(r)$ and $\varphi_{i}(r), i=1,2, \ldots$, respectively. The fission source distribution is given by

$$
P(r)=\int_{4 \pi} \int_{0}^{\infty} v \Sigma_{f}(r, E) \phi(r, \vec{\Omega}, E) d E d \vec{\Omega},
$$

where

$$
\begin{aligned}
\Sigma_{f}(r, E)= & \text { macroscopic fission cross section of neutron } \\
& \text { energy } E \text { at position } r .
\end{aligned}
$$

Here, $k_{\mathrm{i}}$ is defined as the $i$-th eigenvalue of $\lambda$-mode equation of Eq. (1) for a given $\alpha$ value, and is ordered $k_{1}>k_{2} \geq k_{3} \geq$, $\ldots,>0$ where the eigenvalues are assumed to be real and positive. If the $\alpha$ value is equal to $\alpha_{1}$, the fundamental mode eigenvalue of Eq. (1), then $k_{1}=1$. If $\alpha>\alpha_{1}$, then $k_{1}>1$. An operator $A$, which corresponds to a single power iteration, is introduced, then

$$
A \varphi_{i}(r)=k_{i} \varphi_{i}(r) .
$$

Because of completeness, an arbitrary fission source distribution $P(r)$ can be expanded as a linear combination of the eigenfunctions

$$
P(r)=\sum_{i=1}^{\infty} c_{i} \varphi_{i}(r)
$$

where $c_{i}$ is an amplitude of the $i$-th eigenfunction. $P(r)$ is normalized at each iteration throughout the Monte Carlo calculation. For example,

$$
N=\int|P(r)| d r,
$$

where $N$ is the number of particles per iteration. Applying the operator $A$ to $P(r)$ stands for obtaining the fission source distribution in the next iteration:

$$
A P(r)=\sum_{i=1}^{\infty} c_{i} k_{i} \varphi_{i}(r)
$$

Since $k_{1}>k_{2} \geq k_{3} \geq, \ldots,>0$, applying the operator $A$ many times with a constant $\alpha$ value has the limit as:

$$
\lim _{n \rightarrow \infty} \frac{1}{k_{1}^{n}} A^{n} P(r)=c_{1} \varphi_{1}(r)
$$

where only the eigenfunction with the largest eigenvalue survives. Actually, the $\alpha$ value is updated at each iteration by Eq. (7). If $\alpha>\alpha_{1}>0$ and $P(r)$ is assumed to be approximately proportional to the fundamental mode eigenfunction $\varphi_{1}(r)$ after some iterations with the constant $\alpha$ value, then

$$
\sum_{i} v_{p} \Sigma_{f} T_{i} \approx k_{1} N>N
$$

due to $k_{1}>1$. Thus, as can be understood from Eq. (9), $\alpha^{\prime}<$ $\alpha$, which says that the $\alpha$ value does not diverge from the fundamental mode eigenvalue $\alpha_{1}$ corresponding to $k_{1}=1$.

\section{Convergence of Second $\alpha$ Mode Eigenvalue}

Here, a method for obtaining higher order criticality eigenvalues, which was proposed by Booth, ${ }^{4}$ is applied to seeking the second $\alpha$ mode eigenvalue. This method partitions the space into two regions $R_{\mathrm{I}}$ and $R_{\mathrm{II}}$. Two $k_{2}$ 's in the $n$-th iteration are defined as:

$$
k_{2}^{I(n)}=P_{I}^{(n)} / P_{I}^{(n-1)},
$$

and

$$
k_{2}^{I I(n)}=P_{I I}^{(n)} / P_{I I}^{(n-1)},
$$

where

$$
\begin{aligned}
& P_{I}^{(n-1)}=\int_{R_{I}} P^{(n-1)}(r) d r, \\
& P_{I I}^{(n-1)}=\int_{R_{I I}} P^{(n-1)}(r) d r, \\
& P_{I}^{(n)}=\int_{R_{I}} A P^{(n-1)}(r) d r, \\
& P_{I I}^{(n)}=\int_{R_{I I}} A P^{(n-1)}(r) d r,
\end{aligned}
$$

and $P^{(n-1)}(r)$ is the fission source distribution in the $(n-1)$ th iteration. How the space is partitioned into two regions might be arbitrary. However, it is preferable to partition the space according to the sign of $P(r) .{ }^{6}$ ) For example, $P(r)>0$ in $R_{\mathrm{I}}$ and $P(r)<0$ in $R_{\mathrm{II}}$.

The method utilizes the property that $k_{2}^{I(n)}=k_{2}^{I I(n)}$ $=k_{2}$ when $P(r) \propto \varphi_{2}(r)$. When $k_{2}^{I(n)}>k_{2}^{I I(n)}$, this fact means that the component in the region $R_{\mathrm{I}}$ is growing faster than in the region $R_{\mathrm{II}}$ and vice versa. Modifying the fission source distribution to keep two components growing at the same rate leads to suppressing the fundamental mode eigenfunction relative to the second one. This was proved by Booth $^{4)}$ and Yamamoto ${ }^{6}$ for criticality eigenvalue problems. Such a modification of the fission source distribution after each power iteration would be expected eventually to lead to convergence toward the second $\alpha$ mode eigenfunction. Booth proposes that the fission source distribution for the next iteration $P^{(n)}(r)$ be modified as

$$
P^{(n)}(r)=\left(k_{2}^{I I(n)} / k_{2}^{I(n)}\right)^{\gamma} A P^{(n-1)}(r) \text { for } r \in R_{I},
$$

or

$$
P^{(n)}(r)=\left(k_{2}^{I(n)} / k_{2}^{I I(n)}\right)^{\gamma} A P^{(n-1)}(r) \text { for } r \in R_{I I},
$$

where $\gamma>1$. Then, the modified fission source distribution $P^{(n)}(r)$ gets renormalized as in Eq. (15). The modified distribution is used as $P(r)$ in the next cycle. In the case of a criticality eigenvalue problem, the range of $\gamma$ that allows a 


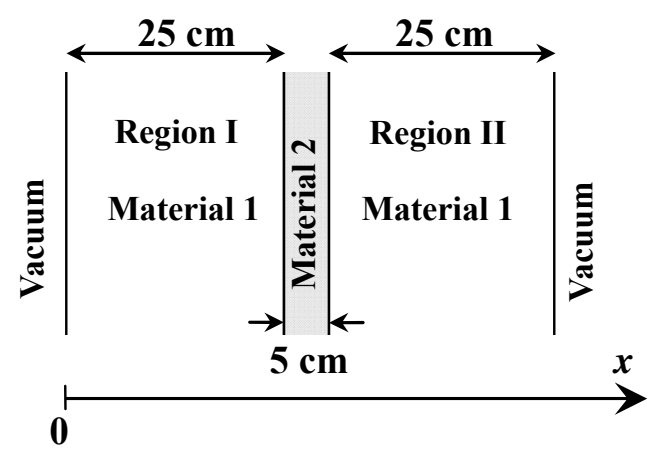

Fig. 3 Calculation model for test problem

convergence toward the second eigenfunction is given by ${ }^{4,6)}$

$$
1<\gamma<\left(k_{1}+k_{2}\right) /\left(k_{1}-k_{2}\right) \text {. }
$$

In the case of an $\alpha$ mode eigenvalue problem, such a range of $\gamma$ for convergence cannot be straightforwardly derived since the $\alpha$ value is updated each iteration. The eigenfunctions change with the $\alpha$ value while the eigenfunctions of a criticality mode eigenvalue problem are fixed throughout the calculation. The range of $\gamma$ for convergence is to be studied empirically in the following section.

\section{Numerical Applications}

\section{Second $\alpha$ Mode Eigenvalue}

The technique for the second $\alpha$ mode eigenvalue that uses Eq. (25) or (26) was applied to a simple test problem in two neutron energy groups. A system for the test problem was composed of two fissionable infinite slabs decoupled by an intervening non-fissionable material. The geometry is symmetric as shown in Fig. 3. The two-energy group constants for the test problem are shown in Table 3. The scattering was isotropic in the laboratory system. The notations in Table 3 are standard. This system is subcritical, and the $k_{\text {eff }}$ without delayed neutrons is 0.958 .

For higher order mode calculations in the Monte Carlo technique, positive and negative weights need to be cancelled during the course of the calculation. The cancellation should be conducted in a pointwise manner. Monte Carlo methods, however, have a difficulty to make such a pointwise cancellation of positive and negative weights. Booth ${ }^{4}$ used a point-detector like technique for the point cancellation. This technique, however, raises a problem how to decide the point positions and the number of points. Furthermore, due to the point-detector like property, it takes long computation time. The computing burden becomes larger in proportion to the square of the number of points used for the pointwise cancellation.

Recently, Booth et al. developed an exact method that cancels positive and negative weights over a region instead of at points or in small discretized bins and has the potential of being significantly more efficient than the other two. ${ }^{13)}$

Here, for the cancellation of positive and negative weights, the geometry of this problem was divided into 200 zones in the fissionable slabs and 40 zones in the intervening slab. The fission reaction rates calculated with the track length
Table 3 Two-group constants for test problem

\begin{tabular}{lcc}
\hline & Material 1 & Material 2 \\
\hline$\Sigma_{t}^{1 *}\left(\mathrm{~cm}^{-1}\right)$ & 0.2 & 0.2 \\
$\Sigma_{t}^{2}\left(\mathrm{~cm}^{-1}\right)$ & 0.4 & 0.4 \\
$\Sigma_{a}^{1}\left(\mathrm{~cm}^{-1}\right)$ & 0.07 & 0.05 \\
$\Sigma_{a}^{2}\left(\mathrm{~cm}^{-1}\right)$ & 0.12 & 0.05 \\
$\Sigma_{f}^{1}\left(\mathrm{~cm}^{-1}\right)$ & 0.02 & 0 \\
$\Sigma_{f}^{2}\left(\mathrm{~cm}^{-1}\right)$ & 0.07 & 0 \\
$\Sigma_{s}^{1 \rightarrow 1}\left(\mathrm{~cm}^{-1}\right)$ & 0.06565 & 0.07575 \\
$\Sigma_{s}^{1 \rightarrow 2}\left(\mathrm{~cm}^{-1}\right)$ & 0.06435 & 0.07425 \\
$\Sigma_{s}^{2 \rightarrow 1}\left(\mathrm{~cm}^{-1}\right)$ & 0.0028 & 0.0035 \\
$\Sigma_{s}^{2 \rightarrow 2}\left(\mathrm{~cm}^{-1}\right)$ & 0.2772 & 0.3465 \\
$v_{\mathrm{p}}$ & 2.5 & - \\
$\chi^{1}$ & 1 & - \\
$\chi^{2}$ & 0 & $3 \times 10^{7}$ \\
$v^{1}(\mathrm{~cm} / \mathrm{s})$ & $3 \times 10^{5}$ & $3 \times 10^{5}$ \\
$v^{2}(\mathrm{~cm} / \mathrm{s})$ & & - \\
\hline
\end{tabular}

* The superscripts means neutron energy group.

estimators were accumulated in the zones. The positive and negative weights of the signed particles were cancelled in the divided zones. At the end of each cycle, the relative fission source intensity in each zone was determined in proportion to the fission reaction rate in the zone. The fission source sites for the next cycle were positioned uniformly within each zone. This cancellation technique is not exact as compared to the pointwise cancellation using the point detector-like technique. The source normalization scheme and cancellation technique that were used in this test problem would not significantly affect calculation results if a zone division is fine enough. Monte Carlo calculations were performed for 2,070 cycles with 20,000 neutrons per cycle with the first 70 cycles skipped. The adjustment parameter in Eq. (10) was set to $\lambda=0$ throughout the calculations of the test problem.

The first $\alpha$ mode eigenfunction and eigenvalue for the forward and adjoint problems were obtained by the technique in Section II. The adjoint eigenfunction was calculated because it is used later for higher order mode calculations. The calculated $\alpha$ values are listed in Table 4 along with the $\alpha$ value calculated by the ONEDANT deterministic discrete ordinates $\operatorname{code}^{14)}$ with the same group constants. The ONEDANT calculation was performed with $\mathrm{S}_{24}$ quadrature and mesh boundaries at $0.25 \mathrm{~cm}$ intervals. Three $\alpha$ values indicate agreement within the statistical uncertainties.

The whole space was partitioned symmetrically into two regions. The left side fissionable material $(0 \leq x \leq 25 \mathrm{~cm})$ 
Table 4 First $\alpha$ mode eigenvalue for test problem

\begin{tabular}{lcc}
\hline & $\alpha\left(\mathrm{s}^{-1}\right)$ & $k_{\mathrm{eff}}$ \\
\hline$\alpha$ value by Monte Carlo & $2155.4 \pm 6.2$ & $1.00006 \pm$ \\
calculation (forward) & & 0.00014 \\
\hline$\alpha$ value by Monte Carlo & $2152.6 \pm 6.5$ & $1.00004 \pm$ \\
calculation (adjoint) & & 0.00019 \\
\hline$\alpha$ value by ONEDANT & 2157.8 & - \\
\hline
\end{tabular}

was assigned to the region $\mathrm{I}$, and the right side $(30 \leq x \leq$ $55 \mathrm{~cm}$ ) to the region II as shown in Fig. 3. The initial starting particles of positive weight +1 were randomly assigned in the region $0 \leq x \leq 25 \mathrm{~cm}$ and $30 \leq x \leq 38 \mathrm{~cm}$. On the other hand, the initial starting particles of negative weight -1 were randomly assigned in the region $38 \leq x \leq 55 \mathrm{~cm}$. The asymmetric distribution of the positive and negative weights was intentionally given for testing the convergence of the second eigenfunction. Following Eqs. (19) through (24), the second eigenvalue $k_{2}^{i}$ in the $i$-th divided region were calculated by:

$$
k_{2}^{i}=\sum_{j} t_{j} v_{p} \Sigma_{f} w_{j} / \sum_{k=1}^{N_{i}} w_{k}, \quad i=\mathrm{I} \text { or II, }
$$

where

$j$ is summed over all trajectories in the $i$-th region, and

$w_{j}=$ particle weight in the $j$-th trajectory (positive or negative),

$t_{j}=\quad$ track length in the $j$-th trajectory,

$N_{i}=\quad$ the number of particles starting from the $i$-th region,

$w_{k}=\quad$ particle weight of the $k$-th starting particle (positive or negative).

The terms in Eq. (7) for obtaining an $\alpha$ value over the whole system were calculated as follows:

$$
\begin{aligned}
& N \equiv \sum_{i=1}^{N_{S}}\left|w_{i}\right|, \\
& \Delta W_{i} \equiv \sum_{m} \sum_{j}\left|\Delta W_{j}^{m}\right|, \\
& \sum_{i} v_{p} \Sigma_{f} T_{i} \equiv \sum_{m} \sum_{j} t_{j}^{m} v_{p} \Sigma_{f}\left|w_{j}^{m}\right|, \\
& \sum_{i} T_{i} / v_{i}(E) \equiv \sum_{m} \sum_{j} t_{j}^{m}\left|w_{j}^{m}\right|,
\end{aligned}
$$

where

$m$ was summed over the all discretization zones, and $j$ was summed over all trajectories in the $m$-th discretization zone, and

$N_{s}=\quad$ number of all starting neutrons in one cycle,

$\Delta W_{j}^{m}=$ weight change in the $j$-th trajectory in the $m$-th discretization zone (positive or negative),

$t_{j}^{m}=\quad$ track length in the $j$-th trajectory in the $m$-th discretization zone,

$w_{j}^{m}=\quad$ particle weight in the $j$-th trajectory in the $m$-th discretization zone (positive or negative).
A method is introduced to assess the convergence of the fission source distribution toward the second eigenfunction. ${ }^{6}$ Auxiliary eigenvectors $\boldsymbol{f}$ and $\boldsymbol{g}$ are defined that are associated with the first eigenfunction and second eigenfunction as follows, respectively:

$$
\begin{aligned}
& \boldsymbol{f}=(1, f)^{t}, \\
& \boldsymbol{g}=(1, g)^{t},
\end{aligned}
$$

where $t$ implies transpose, and

$$
\begin{aligned}
& f=\int_{R_{I I}} \varphi_{1}(r) d r / \int_{R_{I}} \varphi_{1}(r) d r, \\
& g=\int_{R_{I I}} \varphi_{2}(r) d r / \int_{R_{I}} \varphi_{2}(r) d r .
\end{aligned}
$$

It is assumed that $P^{(n)}(r)$ consists of the first eigenfunction $\varphi_{1}(r)$ and second eigenfunction $\varphi_{2}(r)$ and that the eigenfunctions $c_{i} \varphi_{i}(r)$ with $i>2$ already become insignificant after some iterations. That is,

$$
P^{(n)}(r)=c_{1}^{(n)} \varphi_{1}(r)+c_{2}^{(n)} \varphi_{2}(r) .
$$

The amplitudes of $\varphi_{1}(r)$ and $\varphi_{2}(r)$ in the $n$-th iteration, which are denoted by $c_{1}^{(n)}$ and $c_{2}^{(n)}$, respectively, are given by:

$$
\left(\begin{array}{l}
c_{1}^{(n)} \\
c_{2}^{(n)}
\end{array}\right)=\mathbf{U}^{-1}\left(\begin{array}{l}
P_{I}^{(n)} \\
P_{I I}^{(n)}
\end{array}\right),
$$

where

$$
\mathbf{U}^{-1}=\left(\begin{array}{ll}
1 & 1 \\
f & g
\end{array}\right)^{-1}=\frac{1}{g-f}\left(\begin{array}{cc}
g & -1 \\
-f & 1
\end{array}\right) .
$$

A parameter $\left|c_{1}^{(n)} / c_{2}^{(n)}\right|$ becomes smaller as the fission source distribution converges to the second eigenfunction. Thus, the parameter $\left|c_{1}^{(n)} / c_{2}^{(n)}\right|$ is used as a measure for assessing how the second eigenfunction is dominant over the first one and how the convergence to the second eigenfunction is attained.

Since the test problem is symmetrical, it follows that $f=$ 1 and $g=-1$. The transitions of the parameter $c_{1}^{(n)} / c_{2}^{(n)}$ during the initial cycles were calculated for several $\gamma$ values (defined in Eqs. (25) or (26)), and they are shown in Fig. 4. When $\gamma$ is close to unity, e.g., $\gamma=1.2, c_{1}^{(n)} / c_{2}^{(n)}$ becomes zero slowly. As $\gamma$ becomes larger, $c_{1}^{(n)} / c_{2}^{(n)}$ becomes zero earlier. However, significant undershoots of $c_{1}^{(n)} / c_{2}^{(n)}$ are seen for $\gamma=2.8$ and 5.0. If $\gamma$ is larger than around 8.0, the calculation is abnormally terminated due to the large undershoot. Figure 4 illustrates that the second $\alpha$ mode eigenvalue calculations seem to be performed successfully by selecting a $\gamma$ value appropriately. 


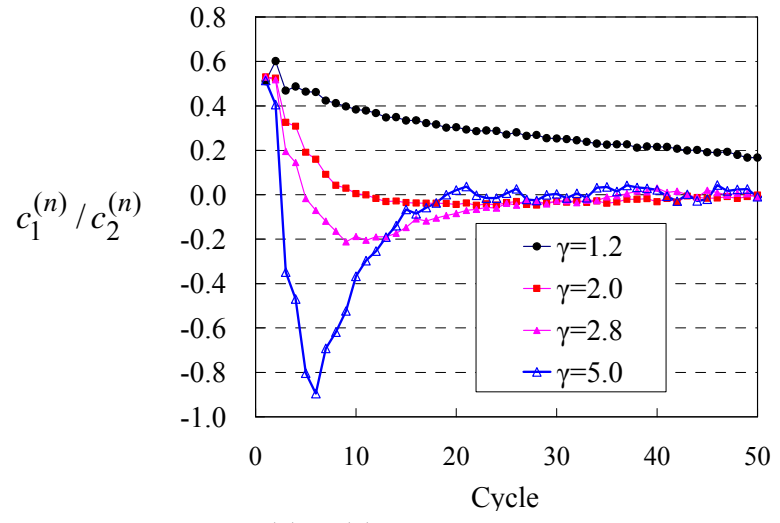

Fig. $4 c_{1}^{(n)} / c_{2}^{(n)}$ transitions for several $\gamma$ s.

The calculated second $\alpha$ eigenvalues for the forward and adjoint problems are listed in Table 5. Since no existing calculation method is available for verification of the second $\alpha$ eigenvalues, the simulation of a pulsed neutron method was performed. Pulsed neutrons in the first energy group were injected at $x=8.0 \mathrm{~cm}$, and then the subsequent neutron counts were accumulated in time bins with the interval of 10 $\mu$ second. The point where the source neutrons were generated was determined so as to excite the second and third eigenfunctions. The time-dependent angular flux distributions $\phi^{g}(r, \boldsymbol{\Omega}, t)$ in the $g$-th energy group can be expanded as a linear combination of the eigenfunctions,

$$
\phi^{g}(r, \mathbf{\Omega}, t)=\sum_{i=1}^{\infty} c_{i}(t) \psi_{i}^{g}(r, \mathbf{\Omega}),
$$

where $c_{i}(t)$ is a time-dependent amplitude of the $i$-th eigenfunction, and $\psi_{i}^{g}(r, \boldsymbol{\Omega})$ is the $i$-th eigenfunction of the forward angular flux in the $g$-th energy group. The forward angular eigenfunction and adjoint angular eigenfunction $\psi_{i}^{*}{ }^{*}(r, \boldsymbol{\Omega})$ satisfy the orthogonal conditions,

$$
\sum_{g=1}^{G} \int_{V} d r \int_{4 \pi} d \mathbf{\Omega} \frac{1}{v_{g}} \psi_{m}^{* g}(r, \mathbf{\Omega}) \psi_{n}^{g}(r, \mathbf{\Omega})=0 \text { for } m \neq n
$$

and

$$
\sum_{g=1}^{G} \int_{V} d r \int_{4 \pi} d \mathbf{\Omega} \frac{1}{v_{g}} \psi_{m}^{* g}(r, \mathbf{\Omega}) \psi_{n}^{g}(r, \mathbf{\Omega}) \neq 0 \text { for } m=n
$$

where $v_{\mathrm{g}}$ is the neutron velocity in the $g$-th energy group. Using Eq. (40) and this orthogonality, the time-dependent amplitude of the $i$-th eigenfunction is given by

$$
c_{i}(t)=\frac{\sum_{g=1}^{G} \int_{V} d r \int_{4 \pi} d \boldsymbol{\Omega} \frac{1}{v_{g}} \psi_{i}^{* g}(r, \boldsymbol{\Omega}) \phi^{g}(r, \mathbf{\Omega}, t)}{\sum_{g=1}^{G} \int_{V} d r \int_{4 \pi} d \mathbf{\Omega} \frac{1}{v_{g}} \psi_{i}^{* g}(r, \boldsymbol{\Omega}) \psi_{i}^{g}(r, \mathbf{\Omega})} .
$$

The time-dependent amplitude of the $i$-th eigenfunction
Table 5 Second $\alpha$ mode eigenvalue for test problem

\begin{tabular}{lcc}
\hline & $\alpha\left(\mathrm{s}^{-1}\right)$ & $k_{\mathrm{eff}}$ \\
\hline $\begin{array}{l}\alpha \text { value by Monte Carlo } \\
\text { calculation (forward) }\end{array}$ & $5703.9 \pm 8.1$ & $\begin{array}{c}1.00012 \pm \\
0.00018\end{array}$ \\
\hline $\begin{array}{l}\alpha \text { value by Monte Carlo } \\
\text { calculation (adjoint) }\end{array}$ & $5726.0 \pm 6.3$ & $\begin{array}{c}1.00004 \pm \\
0.00019\end{array}$ \\
\hline $\begin{array}{l}\text { Pulsed neutron method } \\
\text { simulation }\end{array}$ & $5708.3 \pm 0.8$ & - \\
\hline
\end{tabular}

asymptotically decays with time as follows:

$$
c_{i}(t) \propto e^{-\alpha_{i} t}
$$

where $\alpha_{i}$ is the $i$-th mode eigenvalue. The relative amplitudes of the first and second eigenfunctions are shown in Fig. 5. The second $\alpha$ mode eigenvalue that is listed in the last row in Table 5 was obtained by fitting the time-decay of the amplitude of the second eigenfunction. The second $\alpha$ mode eigenvalues calculated by the $\alpha$ mode eigenvalue calculations indicate good agreement with the one by the pulsed neutron simulation. This agreement constitutes verification of the eigenvalue mode calculation for the second $\alpha$ mode.

\section{Third and Higher $\alpha$ Mode Eigenvalue}

Booth successfully obtained the third and higher criticality eigenvalues by the method that partitions the whole space into several regions without knowing lower order eigenfunctions. ${ }^{4)}$ The author attempted in vain to obtain the third $\alpha$ mode eigenvalue. Then, Hotelling's method, which explicitly subtracts the first and second eigenfunction during the power iteration, was adopted for the third $\alpha$ mode eigenvalue calculation. Suppose that the angular flux $\phi^{g(n)}(r, \boldsymbol{\Omega})$ is obtained after the $n$-th power iteration. The angular flux $\phi^{g(n+1)}(r, \boldsymbol{\Omega})$ used for a starter in the $(n+1)$ th power iteration is obtained by subtracting the first and second $\alpha$ mode eigenfunctions as

$$
\begin{aligned}
& \phi^{g(n+1)}(r, \boldsymbol{\Omega}) \\
& =\phi^{g(n)}(r, \boldsymbol{\Omega})-c_{1}^{(n)} \psi_{1}^{g}(r, \boldsymbol{\Omega})-c_{2}^{(n)} \psi_{2}^{g}(r, \boldsymbol{\Omega})
\end{aligned}
$$

where

$$
c_{i}^{(n)}=\frac{\sum_{g=1}^{G} \int_{V} d r \int_{4 \pi} d \boldsymbol{\Omega} \frac{1}{v_{g}} \psi_{i}^{* g}(r, \mathbf{\Omega}) \phi^{g(n)}(r, \mathbf{\Omega})}{\sum_{g=1}^{G} \int_{V} d r \int_{4 \pi} d \mathbf{\Omega} \frac{1}{v_{g}} \psi_{i}^{* g}(r, \mathbf{\Omega}) \psi_{i}^{g}(r, \mathbf{\Omega})},
$$

This subtraction procedure is also performed for the calculation of the adjoint angular flux. As far as the test problem in this paper is concerned, convergence of the $\alpha$ mode eigenvalue calculations was achieved up to the forth $\alpha$ mode eigenvalue calculation. The calculated third $\alpha$ eigenvalues for the forward and adjoint problems are listed in Table 6. The relative amplitude of the third eigenfunction 


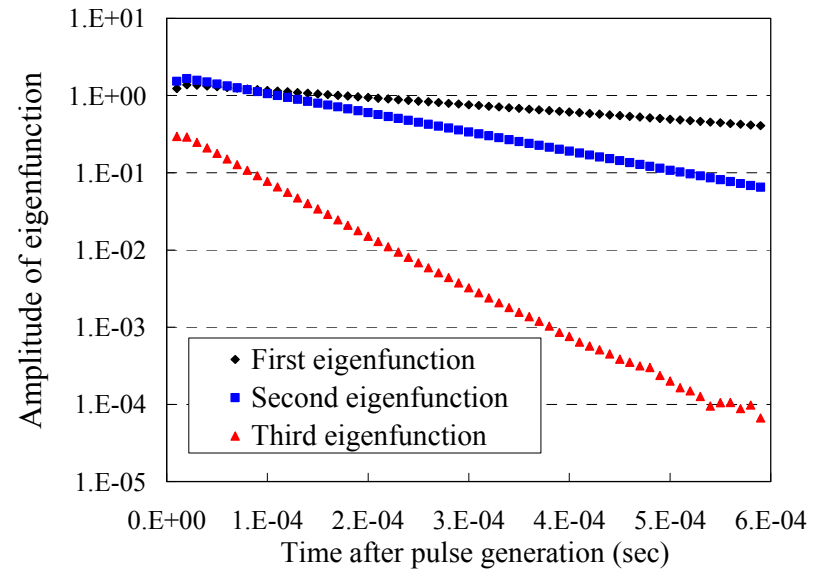

Fig. 5 Time decay of amplitudes of 1-st, 2-nd and 3-rd order

Table 6 Third $\alpha$ mode eigenvalue for test problem

\begin{tabular}{lcc}
\hline & $\alpha\left(\mathrm{s}^{-1}\right)$ & $k_{\text {eff }}$ \\
\hline $\begin{array}{l}\alpha \text { value by Monte Carlo } \\
\text { calculation (forward) }\end{array}$ & $16845 \pm 12$ & $\begin{array}{c}1.00008 \pm \\
0.00022\end{array}$ \\
\hline $\begin{array}{l}\alpha \text { value by Monte Carlo } \\
\text { calculation (adjoint) }\end{array}$ & $16908 \pm 12$ & $\begin{array}{c}1.00033 \pm \\
0.00046\end{array}$ \\
\hline $\begin{array}{l}\text { Pulsed neutron method } \\
\text { simulation }\end{array}$ & $16613 \pm 12$ & - \\
\hline
\end{tabular}

calculated by Eq. (46) is also shown in Fig. 5. The third $\alpha$ mode eigenvalue obtained by fitting the time-decay of the amplitude of the third eigenfunction is listed in Table 6. As time elapses after the pulse generation, the time-dependent flux distributions $\phi^{g}(r, \boldsymbol{\Omega}, t)$ get closer to the first eigenfunction due to the faster decays of higher order eigenfunctions. The amplitude of the third eigenfunction $c_{3}(t)$ approaches zero rapidly, which inevitably makes the calculation of $c_{3}(t)$ inaccurate. However, the agreement between the third $\alpha$ mode eigenvalue by the pulsed neutron method and the one by the $\alpha$ mode eigenvalue calculation may be satisfactory by considering the standard deviations. The calculated fourth $\alpha$ mode eigenvalue was $25,478 \pm 68$ $\left(\mathrm{s}^{-1}\right)$. It was unable to perform the fourth order adjoint calculation and calculations higher than the fourth order with Hotelling's method. Relative forward eigenfunction distributions of the first (i.e., fundamental mode), second, third, and fourth orders are shown in Fig. 6.

\section{Conclusions}

It has been reported in many literatures that $\alpha$ mode eigenvalue calculations in the Monte Carlo technique are difficult for fissionable systems with large subcriticality. Implementing a pseudo absorption term in the neutron transport equation, which was proposed by Yen et al., overcomes the difficulty. The author demonstrated that this technique certainly can provide stable $\alpha$ mode eigenvalue calculations for problems that the original MCNP 4C fails to solve. In addition, the figure of merit of the calculated $\alpha$ value is largely improved by introducing the pseudo absorp-

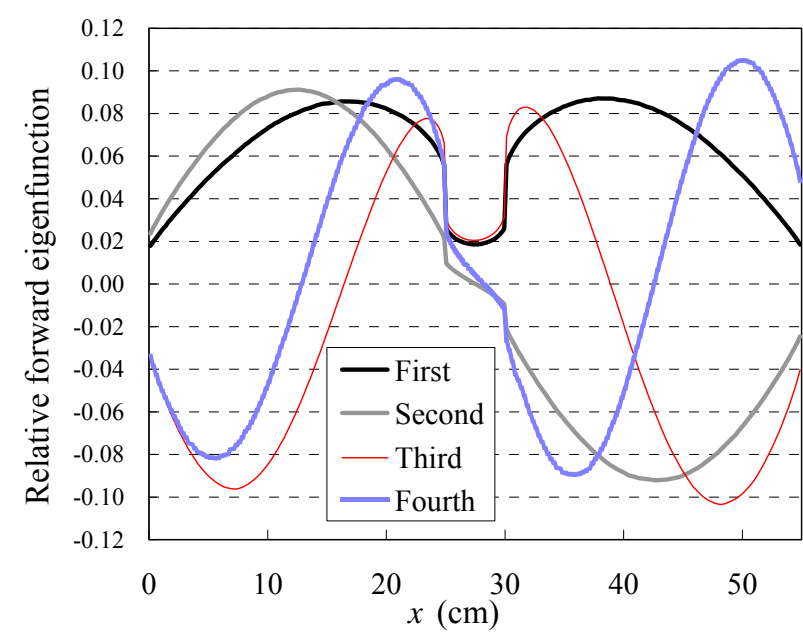

Fig. 6 Eigenfunction distributions of test problem

tion term. However, the $\alpha$ value calculated in a cycle is influenced by the $\alpha$ value used for the calculation when introducing the pseudo absorption term. Thus, if the $\alpha$ value obtained in the previous cycle is used in the next cycle calculation, the variance of the calculated $\alpha$ values would be underestimated. How to estimate an $\alpha$ value used for the next cycle and how to estimate the true standard deviation would be a research issue in the future. The $\alpha$ values calculated by the $\alpha$ mode eigenvalue calculations were verified by comparing the values obtained by the pulsed neutron simulation calculations.

The method that provides eigenfunctions with higher order criticality eigenvalues was applied to higher order $\alpha$ mode eigenvalue calculations. To obtain the second eigenfunction, the method partitions the whole space into two regions. The estimate of the multiplication factor in each region is forced to be equal to each other. The method was found to be applicable to calculations for the second $\alpha$ mode eigenfunction. This paper may be the first attempt to obtain higher order $\alpha$ mode eigenvalues and eigenfunctions by the Monte Carlo technique. The factor $\gamma(>1)$ in Eqs. (25) or (26) needs to be specified in this method. However, the theoretical basis of the appropriate range of the factor $\gamma$ in Eqs. (25) or (26) has not been given in this paper. As it now stands, the factor $\gamma$ has to be determined through a trial and error process.

The numerical examples in this work indicates that the second $\alpha$ eigenvalue calculated by the proposed method was verified by the simulation of the pulsed neutron method. For $\alpha$ mode eigenvalue problem higher than the second order, Hotelling's method was applied. This method explicitly subtracts lower order eigenfunctions from the fission source distribution. The third and fourth order forward eigenfunctions of the test problem were successfully obtained with the use of Hotelling's method.

Like higher order criticality calculations, higher order $\alpha$ mode eigenvalue calculations in the Monte Carlo method requires the cancellation of positive and negative weights of particles. This work adopted the space discretization technique for the cancellation of the signed particles as an 
alternative. Recently, an exact method for canceling positive and negative particle weights has been developed by Booth et al. The adoption of the new method would be promising for higher order mode calculations by the Monte Carlo method.

\section{References}

1) D. Brockway, P. Soran, P. Whalen, "Monte Carlo $\alpha$ calculation," LA-UR-85-1224, Los Alamos National Laboratory (LANL) (1985).

2) J. F. Briesmeister, (Ed.), "MCNP-A general Monte Carlo N-particle transport code, version 4C," LA-13709-M, Los Alamos National Laboratory (LANL) (2000).

3) T. Endo, Y. Yamane, A. Yamamoto, "Space and energy dependent theoretical formula for the third order neutron correlation technique," Ann. Nucl. Energy, 33, 521-537 (2006).

4) T. E. Booth, "Computing the higher k-eigenfunctions by Monte Carlo power iteration: a conjecture," Nucl. Sci. Eng., 143, 291-300 (2003).

5) T. E. Booth, "Power iteration method for the several largest eigenvalues and eigenfunctions," Nucl. Sci. Eng., 154, 48-62 (2006).

6) T. Yamamoto, "Convergence of the second eigenfunction in Monte Carlo power iteration," Ann. Nucl. Energy, 36, 7-14 (2009).

7) T. Yen, C. Chen, W. Sun, B. Zhang, D. Tian, "Prompt time constants of a reflected reactor," T. Fukahori (Ed.), Proc. 2006
Symposium on Nuclear Data, Tokai-mura, Ibaraki-ken, Japan, Jan. 25-26, 2007, Nuclear Data Division, Atomic Energy Society of Japan (2007), [CD-ROM].

8) T. Yamamoto, Y. Miyoshi, "An algorithm of $\alpha$ - and $\gamma$-mode eigenvalue calculations by Monte Carlo method," Proc. 7th Int. Conf. on Nuclear Criticality Safety, ICNC 2003, Tokai-mura, Ibaraki, Japan, Oct. 20-24, 2003, JAERI-Conf 2003-019, Japan Atomic Energy Research Institute (JAERI) (2003).

9) D. E. Kornreich, D. K. Parsons, "Time-eigenvalue calculations in multi-region Cartesian geometry using Green's function," Ann. Nucl. Energy, 32, 964-985 (2005).

10) T. Yamamoto, Y. Miyoshi, T. Kiyosumi, "Validating JENDL-3.3 for water-reflected low-enriched uranium solution systems using STACY ICSBEP benchmark models," Nucl. Sci. Eng., 145, 132-144 (2003).

11) H. Yoshikawa, J. Wakabayashi, "An approximate calculation method of the $\lambda, \omega_{p}, \omega_{d}$ eigenvalue problem of the group diffusion equation," J. Nucl. Sci. Technol., 7[7], 355-365 (1970).

12) K. Hashimoto, K. Nishina, "Calculations of spatial harmonics in two-dimensional multiplicative systems," J. Atomic Energy Soc. Japan, 33[9], 882-889 (1991), [in Japanese].

13) T. E. Booth, J. E. Gubernatis, "Exact regional Monte Carlo weight cancellation for second eigenfunction calculations," Nucl. Sci. Eng., 165, 283-291 (2010).

14) R. E. Alcouffe, R. S. Baker, F. W. Brinkley, D. R. Marr, R. D. O'Dell, W. F. Walters, "DANTSYS: A diffusion accelerated neutral particle transport code system," LA-12969-M, Los Alamos National Laboratory (LANL) (1995). 